Pacific Journal of Mathematics

A REMARK ON THE SYMMETRY OF SOLUTIONS TO 


\title{
A REMARK ON THE SYMMETRY OF SOLUTIONS TO NONLINEAR ELLIPTIC EQUATIONS
}

\author{
JI MIN
}

This note gives a necessary and sufficient condition for solutions of second order elliptic equations to be radially symmetric.

\section{Introduction.}

1.1. In an elegant paper [GNN], Gidas-Ni-Nirenberg proved that the positive solutions of

$$
\begin{cases}\Delta u=f(u) & \text { in } B, \\ u=0 & \text { on } \partial B, \\ u \in C^{2}(\bar{B}), & \end{cases}
$$

must be radially symmetric. Here $f$ is $C^{1}$ and $B$ is the $n$-dimensional ball: $\left\{x \in R^{n} ;|x|<1\right\}$. Obviously a symmetric solution of (1) is not necessary to be positive. In this note, we give a necessary and sufficient condition for symmetric solutions of (1). The main result is the following

THEOREM 1. Suppose $n \geq 2$. A solution $u$ of $(1)$ is radially symmetric if and only if its nodal set $\{x \in \bar{B} ; u(x)=0\}$ is radially symmetric.

REMARK. It is interesting to note that Theorem 1 need not hold in case $n=1$. For, $u=\sin x$ solves

$$
u^{\prime \prime}=-u \text { in }[-\pi, \pi]
$$

with the symmetric nodal set $\{0\} \cup\{-\pi, \pi\}$, but $u$ is not radially symmetric since $\sin (-x)=-\sin x$.

It is clear that the result of [GNN] is a special case of Theorem 1 since the nodal set of a positive solution to (1) is the sphere $\partial B$.

In order to prove Theorem 1, we need the following two preliminary results.

THEOREM 2. Let $u \in C^{2}(\bar{B})$ satisfy

$$
\Delta u=f(u) \text { in } B \text {. }
$$


If the nodal set of $u$ consists of spheres with the center 0 , then these spheres must be isolated unless $u \equiv 0$.

THEOREM 3. Let $n \geq 2$ and $u \in C^{2}(\bar{B})$ satisfy

$$
\begin{cases}\Delta u=f(u) & \text { in } B \\ u>0 & \text { in } B \backslash\{0\}, \\ u=0 & \text { on } \partial B\end{cases}
$$

Then $u>0$ in $B$.

REMARK. In case $n=1$, Theorem 3 need not hold. For example, let $u(x)=\sin \left(x-\frac{\pi}{2}\right)+1$ for $x \in[-2 \pi, 2 \pi]$, we have

$$
\begin{cases}u^{\prime \prime}=1-u & \text { in }(-2 \pi, 2 \pi), \\ u>0 & \text { in }(-2 \pi, 2 \pi) \backslash\{0\}, \\ u=0 & \text { at } x=0,-2 \pi, 2 \pi\end{cases}
$$

1.2. The proof of Theorem 3 is based on Lemma 12.1 in [GNN], we rewrite it in the form.

Lemma A. Let $p=\left(p^{1}, p^{2}, \ldots, p^{n}\right) \in \partial B$ with $p^{1}>0$. Assume for some $\varepsilon>0$ that $u$ is a $C^{2}$ function satisfying equation (2) in $\bar{\Omega}_{\varepsilon}$ where $\Omega_{\varepsilon}=B \cap\{x ;|x-p|<\varepsilon\}, u>0$ in $\bar{\Omega}_{\varepsilon} \backslash \partial B \cap\{x ;|x-p|<\varepsilon\}$ and $u=0$ on $\partial B \cap\{x ;|x-p|<\varepsilon\}$. Then there exists $\delta>0$ such that in $B \cap\{x ;|x-p|<\delta\}, \frac{\partial u}{\partial x_{1}}<0$.

\section{Proofs.}

2.1. Proof of Theorem 2. We may assume that the nodal set of $u$ is $\bigcup_{\lambda \in \Lambda} S(\lambda)$ where $\Lambda \subset[0,1]$ and $S(\lambda)=\left\{x \in R^{n} ;|x|=\lambda\right\}$. It needs to be proved that the set $\Lambda$ contains only isolated points unless $u \equiv 0$. Suppose that there is a sequence $\left\{\lambda_{i}\right\} \subset \Lambda$ with $\lambda_{i} \rightarrow \bar{\lambda}$. Using the polar coordinates $x=r \xi$ where $\xi \in S^{n-1}$ and $r^{2}=x_{1}^{2}+x_{2}^{2}+\cdots+x_{n}^{2}$, we obtain that $u=\frac{\partial u}{\partial r}=\frac{\partial^{2} u}{\partial r^{2}}=0$ for $r=\bar{\lambda}$, which implies that

$$
u(0)=\frac{\partial u}{\partial x_{i}}(0)=\frac{\partial^{2} u}{\partial x_{l}^{2}}(0)=0 \quad(l=1,2, \ldots, n)
$$

when $\bar{\lambda}=0$, and that $u=D_{\xi} u=D_{\xi}^{2} u=0$ on $S(\bar{\lambda})$ when $\bar{\lambda}>0$. Thus, in both cases, $u=\Delta u=0$ on $S(\bar{\lambda})$, and, from (2) we conclude that $f(0)=0$. Set

$$
c(x)=\int_{0}^{1} f^{\prime}(t u(x)) d t
$$


In case $\bar{\lambda}>0$, we have

$$
\begin{cases}\Delta u-c(x) u=0 & \text { in }\{x ;|x|<\bar{\lambda}\}, \\ u=\frac{\partial u}{\partial r}=0 & \text { on } S(\bar{\lambda}),\end{cases}
$$

and obtain $u=0$ in $B$ by uniqueness of solutions to Cauchy's problem of linear elliptic equations. Now it remains to consider the case $\bar{\lambda}=0$. Set

$$
w(x)=\cos N x_{1} \cdot \cos N x_{2} \cdots \cdot \cos N x_{n},
$$

where $N$ is taken to be large enough so that

$$
c(x)+N^{2} \geq 0 .
$$

Put $u=w \cdot v$ for $|x|<\frac{\pi}{2 N}$. It is easy to see that

$$
\left\{\begin{array}{l}
\Delta w=-N^{2} w \\
w>0
\end{array} \text { in }\left\{x ;|x|<\frac{\pi}{2 N}\right\}\right.
$$

and $S\left(\lambda_{i}\right) \subset\left\{x ;|x|<\frac{\pi}{2 N}\right\}$ for $i$ large enough since $\lambda_{i} \rightarrow 0$ as $i \rightarrow \infty$. On account of $(2)$, it follows

$$
\begin{cases}\Delta v+\frac{\nabla W}{W} \nabla v-\left(c(x)+N^{2}\right) v=0 & \text { in }\left\{x ;|x|<\lambda_{i}\right\}, \\ v=0 & \text { on } S\left(\lambda_{i}\right) .\end{cases}
$$

Because of (4), a well-known maximum principle for second order linear elliptic equations can be applied, and that $v=0$ is obtained, so $u=0$ for $|x|<\lambda_{i}$, and in turn $u=0$ in $B$. The proof is completed.

2.2. Proof of Theorem 3. Suppose for contradiction that $u(0)=0$. Automatically $\nabla u(0)=0$. For $0 \leq \lambda<1$, denote $\Sigma_{\lambda}=\{x \in B$; $\left.x_{1}>\lambda\right\} ; T_{\lambda}=\left\{x \in B ; x_{1}=\lambda\right\}$, and for $x \in \Sigma_{\lambda}$, denote by $x^{\lambda}$ the reflexion of $x$ with respect to $T_{\lambda}$, denote by $\Sigma_{\lambda}^{\prime}$ the reflexion of $\Sigma_{\lambda}$ with respect to $T_{\lambda}$. Set

$$
\Lambda=\left\{\lambda \in(0,1) ; u\left(x^{\lambda}\right)>u(x) \text { in } \Sigma_{\lambda}, \frac{\partial u}{\partial x_{1}}<0 \text { on } T_{\lambda}\right\},
$$

which is not empty by Lemma $A$ and a similar argument to [GNN]. First of all we prove inf $\Lambda \in \Lambda$. Indeed, there holds

$$
\begin{cases}u\left(x^{\alpha}\right) \geq u(x) & \text { in } \Sigma_{\alpha} \\ \frac{\partial u}{\partial x_{1}} \geq 0 & \text { on } T_{\alpha}\end{cases}
$$


where $\alpha=\inf \Lambda$. Letting $w(x)=u\left(x^{\alpha}\right)$ for $x \in \Sigma_{\alpha}$ and

$$
c(x)=\int_{0}^{1} f^{\prime}(u+t(w-u)) d t
$$

we have

$$
\left\{\begin{array}{l}
\Delta(w-u)-c(x)(w-u)=0 \\
(w-u) \geq 0 \text { in } \Sigma_{\alpha} \\
(w-u)=0 \text { on } T_{\alpha} .
\end{array}\right.
$$

Then for $K>0$,

$$
\Delta(w-u)-(K+c(x)) \cdot(w-u)=-K(w-u) \leq 0 \text { in } \Sigma_{\alpha} .
$$

Taking $K$ large enough, we may apply the Hopf maximum principle to $(w-u)$ and obtain that either

$$
(w-u)=0 \quad \text { in } \Sigma_{\alpha}
$$

or

$$
\left\{\begin{array}{l}
w(x)>u(x) \quad \text { in } \Sigma_{\alpha}, \\
\frac{\partial}{\partial \bar{n}}(w-u)(p)<0,
\end{array}\right.
$$

where $p \in \partial \Sigma_{\alpha}$ such that $(w-u)(p)=0$ and $\bar{n}=\bar{n}(p)$ is the outward normal vector of $\partial \Sigma_{\alpha}$ at $p$. Then (5) cannot hold since $n \geq 2$ and $u=0$ on $\partial B ; u>0$ in $B \backslash\{0\}$. Now (6) holds, then $u\left(x^{\alpha}\right)>u(x)$ in $\Sigma_{\alpha}$, and on $T_{\alpha}$,

$$
2 \frac{\partial u}{\partial x_{1}}=\frac{\partial}{\partial\left(-x_{1}\right)}(w-u)<0
$$

since $(w-u)=0$, which means $\alpha \in \Lambda$. Next it is easy to see that $\alpha \geq \frac{1}{2}$. If $\alpha=\frac{1}{2}$, let $p_{0}=(1,0, \ldots, 0) \in \partial B$, then $p_{0}^{\alpha}=0$, and

$$
(w-u)\left(p_{0}\right)=u\left(p_{0}^{\alpha}\right)-u\left(p_{0}\right)=0 .
$$

By (6) we have

$$
\frac{\partial}{\partial x_{1}}(w-u)\left(p_{0}\right)<0, \quad \text { i.e. }-\frac{\partial u}{\partial x_{1}}(0)-\frac{\partial u}{\partial x_{1}}\left(p_{0}\right)<0 \text {. }
$$

Then we get

$$
\frac{\partial u}{\partial x_{1}}(0)>-\frac{\partial u}{\partial x_{1}}\left(p_{0}\right) \geq 0
$$

a contradiction since $\nabla u(0)=0$. Thus $\alpha>\frac{1}{2}$. In this case we claim that there exists $\alpha_{0}<\alpha$ such that $\alpha_{0} \in \Lambda$, which will contradict the assumption $\alpha=\inf \Lambda$ and our proof would then be completed. To 
this end, we assume again for contradiction that there exists a sequence $\left\{\alpha_{i}\right\}$ with $\alpha_{i} \rightarrow \alpha$ but $\alpha_{i} \notin \Lambda$ which means that either

$$
u\left(a_{i}^{\alpha}\right) \leq u\left(a_{i}\right) \text { for some } a_{i} \in \Sigma_{\alpha_{i}}
$$

or

$$
\frac{\partial u}{\partial x_{1}}\left(b_{i}\right) \geq 0 \text { for some } b_{i} \in T_{\alpha_{i}} .
$$

The latter cannot always remain true for any subsequence of $\{i\}$ since, otherwise, it implies that $\frac{\partial u}{\partial x_{1}} \geq 0$ at some point on $T_{\alpha}$ when $\left\{b_{i}\right\}$ do not approach $\partial B$, contradicting $\alpha \in \Lambda$, and that there exists a point in any neighborhood of $b$ such that $\frac{\partial u}{\partial x_{1}} \geq 0$ when $b_{i} \rightarrow b \in \partial B$, contradicting Lemma A since $b=\left(b^{1}, \ldots, b^{n}\right)$ with $b^{1}=\alpha>0$. Now let $a_{i} \rightarrow \bar{a} \in \bar{\Sigma}_{\alpha}$. From (7) $u\left(\bar{a}^{\alpha}\right) \leq u(\bar{a})$, and $\bar{a} \in \partial \Sigma_{\alpha}$ by $\alpha \in \Lambda$. But because $\alpha>\frac{1}{2}$, for $x \in \partial \Sigma_{\alpha} \backslash \bar{T}_{\alpha} \subset \partial B$, where $\bar{T}_{\alpha}$ is the closure of $T_{\alpha}$, obviously $u\left(x^{\alpha}\right)>0=u(x)$. Thus we further have $\bar{a} \in \bar{T}_{\alpha}$. Let $L_{i}$ be the segment joining $a_{i}^{\alpha_{i}}$ and $a_{i}$, having $(1,0, \ldots, 0)$ as the tangent vector. From (7) it is seen that there exists $y_{i} \in L_{i}$ such that $\frac{\partial u}{\partial x_{1}}\left(y_{i}\right) \geq 0$. Since $\bar{a} \in \bar{T}_{\alpha}, y_{i}$ must also tend to $\bar{a}$. And automatically $\frac{\partial u}{\partial x_{1}}(\bar{a}) \geq 0$, which leads to a contradiction when $\bar{a} \in T_{\alpha}$. Then $\bar{a} \in \partial \bar{T}_{\alpha} \subset \partial B$. But we have seen that $\frac{\partial u}{\partial x_{1}}\left(y_{i}\right) \geq$ 0 and $y_{i} \rightarrow \bar{a}$, which contradicts Lemma A. Thus we complete the proof.

2.3. Proof of Theorem 1. Denote $B(\lambda)=\left\{x \in R^{n} ;|x|<\lambda\right\}$. The necessity is obvious. For sufficiency, by Theorem 2 , the nodal set of $u$ must be $\bigcup_{i=1}^{k} S\left(\lambda_{i}\right)$ where $0 \leq \lambda_{1}<\lambda_{2}<\cdots<\lambda_{k}=1$. We further prove $\lambda_{1}>0$.

Indeed suppose $\lambda_{1}=0$, i.e. $u(0)=0$. We see that there are no nodal points of $u$ in $B\left(\lambda_{2}\right) \backslash\{0\}$, which, together with the fact that $B\left(\lambda_{2}\right) \backslash\{0\}$ is path-connected (since $n \geq 2$ ), implies that $u$ is positive (or negative) in $B\left(\lambda_{2}\right) \backslash\{0\}$. Then from Theorem 3 we have $u(0)>0$ (or $u(0)<0$ ) also. It contradicts $u(0)=0$, which shows $\lambda_{1}>0$.

Now in $B\left(\lambda_{1}\right), u$ is positive (or negative). It allows us to apply the result of [GNN] to conclude that $u$ is radially symmetric in $B\left(\lambda_{1}\right)$. It is clear that

$$
\frac{\partial u}{\partial r}=\text { const. } \quad \text { on } S\left(\lambda_{1}\right) .
$$

Let $T: R^{n} \rightarrow R^{n}$ be any rotation transform. Since equation (2) is invariant under the transform $T, v=u(T x)$ also solves (2). On 
$S\left(\lambda_{1}\right)$, obviously $v=u$, and $\frac{\partial v}{\partial r}=\frac{\partial u}{\partial r}$ by (9). Then $(v-u)$ is a solution to the Cauchy problem

$$
\begin{aligned}
\Delta w & =\left(\int_{0}^{1} f^{\prime}(t v+(1-t) u) d t\right) \cdot w \text { in } B, \\
w & =\frac{\partial w}{\partial r}=0 \quad \text { on } S\left(\lambda_{1}\right)
\end{aligned}
$$

and constantly equals 0 by the uniqueness of the Cauchy problem, i.e. $u(x)=u(T x)$ in $B$ for any rotation transforms $T$, which means $u$ is radially symmetric in $B$. We finish the proof of our main theorem.

Acknowledgment. It is a pleasure to thank Professor W.-M. Ni for helpful discussions.

\section{REFERENCES}

[GNN] B. Gidus, W.-M. Ni and L. Nirenberg, Symmetry and related properties via the maximum principle, Comm. Math. Phys., 68 No. 3, (1979), 209-243.

Received July 30, 1990.

International Center for Theoretical Physics

TRIESTE, ITALY 


\section{PACIFIC JOURNAL OF MATHEMATICS EDITORS}

V. S. VARADARAJAN

(Managing Editor)

University of California

Los Angeles, CA 90024-1555-05

Herbert Clemens

University of Utah

Salt Lake City, UT 84112

F. Michael Christ

University of California

Los Angeles, CA 90024-1555

THOMAS ENRIGHT

University of California, San Diego

La Jolla, CA 92093

\section{C. MOORE}

University of California

Berkeley, CA 94720

MARTIN SCHARLEMANN

University of California

Santa Barbara, CA 93106

HAROLD STARK

University of California, San Diego

La Jolla, CA 92093

STEVEN KERCKHOFF

Stanford University

Stanford, CA 94305

ASSOCIATE EDITORS

R. ARENS

E. F. BECKENBACH (1906-1982)

B. H. NeumanN

F. WOLF

(1904-1989)

K. YosHIDA

SUPPORTING INSTITUTIONS

UNIVERSITY OF ARIZONA

UNIVERSITY OF OREGON

UNIVERSITY OF BRITISH COLUMBIA

UNIVERSITY OF SOUTHERN CALIFORNIA

CALIFORNIA INSTITUTE OF TECHNOLOGY

UNIVERSITY OF CALIFORNIA

MONTANA STATE UNIVERSITY

STANFORD UNIVERSITY

UNIVERSITY OF NEVADA, RENO

UNIVERSITY OF HAWAII

NEW MEXICO STATE UNIVERSITY

OREGON STATE UNIVERSITY

UNIVERSITY OF UTAH

WASHINGTON STATE UNIVERSITY

UNIVERSITY OF WASHINGTON 


\section{Pacific Journal of Mathematics}

\section{Vol. 153, No. $1 \quad$ March, 1992}

Patrick Robert Ahern and Carmen Cascante, Exceptional sets for Poisson integrals of potentials on the unit sphere in $\mathbf{C}^{n}, p \leq 1 \ldots \ldots \ldots \ldots \ldots$

David Peter Blecher, The standard dual of an operator space ........... 15

Patrick Gilmer, Real algebraic curves and link cobordism .............. 31

Simon M. Goberstein, On orthodox semigroups determined by their

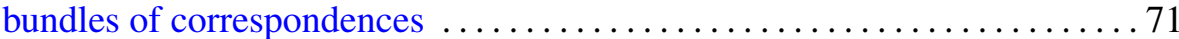

John Kalliongis and Darryl John McCullough, Homeotopy groups of irreducible 3-manifolds which may contain two-sided projective planes .......................................... 85

Yuji Konishi, Masaru Nagisa and Yasuo Watatani, Some remarks on actions of compact matrix quantum groups on $C^{*}$-algebras $\ldots \ldots \ldots \ldots 119$

Guojun Liao and Luen-Fai Tam, On the heat equation for harmonic maps from noncompact manifolds ........................... 129

John Marafino, Boundary behavior of a conformal mapping . .......... 147

Ji Min, A remark on the symmetry of solutions to nonlinear elliptic

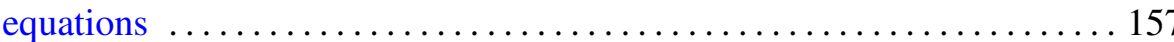

Paul Nevai and Walter Van Assche, Compact perturbations of orthogonal

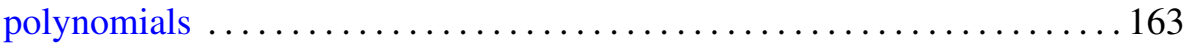

Kyril Tintarev, Level set maxima and quasilinear elliptic problems ....... 185 